\title{
Effect of the shape of wet wood particles on ignition characteristics
}

\author{
Samen Syrodoy ${ }^{1, *}$, Ksenia Bugaeva ${ }^{1}$ \\ ${ }^{1}$ National Research Tomsk Polytechnic University, 634050 Tomsk, Russia
}

\begin{abstract}
The results of experimental studies of various geometric shape wood biomass single particles ignition processes (on the example of a cylinder and a cube) are presented. According to the results of the experiments, it has been stated that the shape of the fuel particle can influence the characteristics and conditions of ignition, but it is insignificant. It has been demonstrated, that in most cases cylindrical particles ignite faster than cubic particles. However, in this respect, two ignition modes are set: high temperature (the cube fires up faster) and low temperature (the cylinder ignites faster).
\end{abstract}

\section{Introduction}

In recent years, wood biomass has become increasingly attractive as a fuel for thermal power plants [1-3]. This trend persists not only in the United States, but also in Eastern Europe [4]. For example, the most modern thermal power plant Avedore (Denmark) [5, 6] completely proceded to the burning of wood pellets in the second half of 2016 . The latter is explained by the number of undoubted advantages of wood fuel in comparison with traditional types of solid (coal), liquid (fuel oil) and gaseous (natural gas) types of energy carriers [7,8]. First of all, wood is the only renewable fuel [7] in many regions of the North and (especially) South America, Asia and Africa. It should also be noted that when burning wood pellets much less (5 times) oxides of nitrogen and sulfur are formed [9] (compared to coal). It is also important that woodworking industry waste can be used as a raw material for the production of fuel based on wood [10]. The storage of the latter causes great problems due to their high fire hazard. For this reason, the most expedient is the use of such wood as a solid fuel for small-sized boiler units (and, in the long term, for large thermal power plants). However, the theory allowing to conduct predictive modeling of the conditions and characteristics of even dry small, medium and large wood particles ignition in the high-temperature gases flow hasn't been developed yet. The latter is due to the fact that the specific properties of wood fuel (the type of wood, external and internal moisture, the anisotropy of wood structure, etc.) have a significant effect on the conditions of ignition. One of the most important parameters is the shape of the particle. As is known from the theory of heat conduction [11], the shape of the body can have a significant effect on the heat transfer characteristics. Accordingly, it can reasonably be assumed that the characteristics of wood particles ignition, for example cube- or cylinder-shaped, will differ

${ }^{*}$ Corresponding author: syrodoy@tpu.ru 
substantially. The currently known studies [12] of the effect of the geometry of fuel particles on the dynamics of their ignition have been carried out for coal-based fuel compositions. At the same time, the questions of the difference in the characteristics of spherical, cylindrical and cubic shape wood particles ignition have not been given sufficient attention. However, we can say that the optimization of the shapes of fuel briquettes made of wood-chip waste is an urgent task for the energy industry. For this reason, the aim of the work is an experimental study of the processes of thermal preparation and of various geometric shapes moist wood particles ignition.

\section{Experiment}

Experimental studies were carried out at a stand similar in its methods of recording and processing experimental data [13]. A particle of wood was fixed on a ceramic holder and brought by a remote-controlled coordinate device into a hollow ceramic cylinder whose inner surface was heated to the temperature of $1270 \mathrm{~K}$. The registration of the processes of heat and mass transfer and ignition of the fuel particle was carried out by a high-speed Photron FASTCAM CA4 5 video camera (video speed up to 20,000 fps). The air temperature inside the cylinder $\left(\mathrm{T}_{\mathrm{e}}\right)$ in experiments varied over a fairly wide range (from $870 \mathrm{~K}$ to $1270 \mathrm{~K}$ ). The period of time from the start of the heat exposure (the moment of entering the cylinder cavity) to the ignition (the appearance of a flame) was considered to be the ignition delay time $\left(\mathrm{t}_{\mathrm{i}}\right)$. The particle sizes varied in the range of $1<\delta<3 \cdot 10-3 \mathrm{~m}$. The systematic error in determining the main measured parameters $\left(\mathrm{Te}, \mathrm{t}_{\mathrm{i}}\right)$ was no more than $5 \%$. The confidence interval for determining tign at a confidence probability of 0.95 did not exceed $17 \%$.

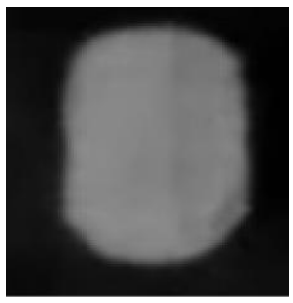

$\mathrm{t}=0 \mathrm{~s}$.

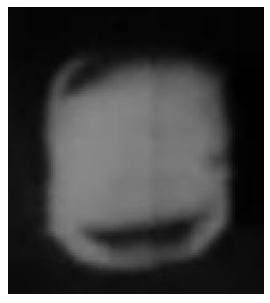

$\mathrm{t}=1.75 \mathrm{~s}$.

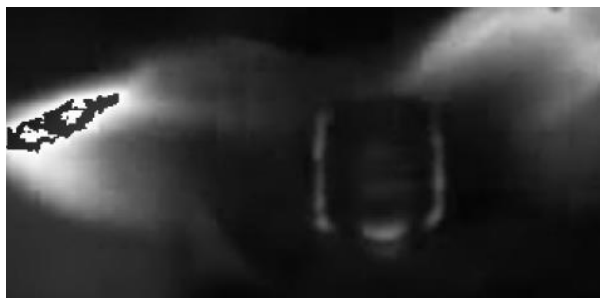

$\mathrm{t}=2.35 \mathrm{~s}$.

Fig.1. Frames of a typical videogram of a moist wood cylindrical particle ignition processes.

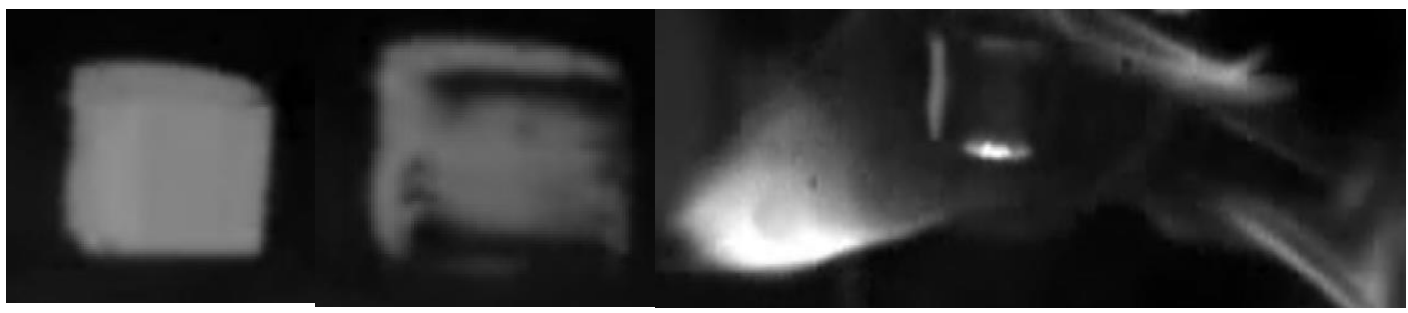

$\mathrm{t}=0 \mathrm{~s}$.

$\mathrm{t}=1.85 \mathrm{~s}$.

$\mathrm{t}=2.75 \mathrm{~s}$.

Fig.2. Frames of a typical videogram of a moist wood cubic particle ignition processes.

\section{The results}

Fig. 1 shows the frames of a typical videogram of a moist wood cylindrical particle ignition processes. (diameter $\delta=3 \cdot 10^{-3} \mathrm{~m}$ ). The temperature of the external environment is $\mathrm{Te}=$ $1273 \mathrm{~K}$. The total time from the start of heating to the moment of ignition is $t_{i} \approx 2.35 \mathrm{~s}$. As 
experiments show, the process of thermal preparation can be conditionally divided into a number of successive interrelated stages (inert heating, water evaporation, thermal decomposition of the organic part of the fuel, ignition of volatiles, ignition of carbon). At the initial moment (frame a), a particle of wood enters a high-temperature environment. Inert heating initiates the water evaporation and thermal decomposition (frame b). A detailed (at a great zooming) analysis of frame b shows the characteristic darkening of the particle surface. In $\mathrm{t}=2.35 \mathrm{~s}$ after the start of heating, the ignition of the volatiles occurs (frame c). It should be noted that a torch of burning volatiles is formed, whose axis direction corresponds to the direction of the wood fibers.

Fig. 2 shows typical frames of the videogram of the processes of moist wood cubic particles ignition. Having analyzed the frames, we can say that the main stages of thermal preparation are similar to Figure 1. It should be noted that the thermal decomposition process is localized mainly at the corners of the cube. The latter is explained by the large heat flows in these places. Also, the analysis of the frames of the videogram shows that when the volatiles are ignited, a torch is formed, whose axis direction coincides with the direction of the wood fibers. Accordingly, it can be said that the characteristics of the ignition of a particle essentially depend on the anisotropy of the thermophysical and filtration properties of the wood.

Figure 3 shows the dependences of the ignition delay times for moist wood particles of different shapes (cylinder and cube). The dependence analysis shows that the particle shape can have an effect on ignition characteristics. However, the degree of such influence is insignificant. It should be noted that the existence of two modes of ignition is possible: high-temperature and low-temperature with the conventional border of $\mathrm{T}_{\mathrm{e}} \approx 973 \mathrm{~K}$. Under $\mathrm{T}_{\mathrm{e}}<973 \mathrm{~K}$, cubic particles ignite faster than cylindrical ones, at higher temperatures of the external environment, cylindrical fuel particles ignite earlier than cubic ones. The latter is most likely due to the fact that when the cylinder is heated, the diffusing volatiles form a uniformly distributed vapor-gas mixture, which is ignited at critical temperatures. Under conditions of low-temperature heating, heterogeneous ignition occurs as a rule. For this reason, the cubic particles ignite faster because of the concentration of heat flows in the zones close to the corner points of the cube.

\section{Conclusion}

According to the results of experimental studies, it has been stated that the shape of a particle of wood fuel can influence the ignition characteristics, but this effect is insignificant. It is shown that when igniting a volatile, the direction of its axis coincides

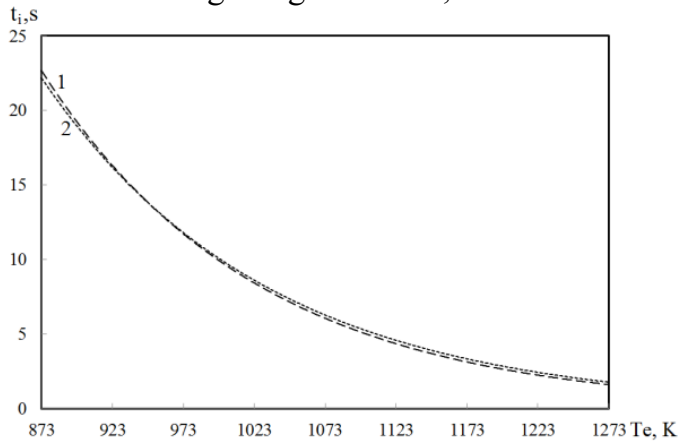

Fig. 3. Dependence of ignition delay on the temperature of the external environment for wood particles of various shapes: 1 - cylinder; 2 - cube. 
with the direction of the fibers of the wood. Accordingly, it can be assumed that the characteristics of ignition substantially depend on the thermal and filtration anisotropy of the wood. According to the results of the experiments, two ignition modes are distinguished: high-temperature (Te $>973 \mathrm{~K})$ and low-temperature $(\mathrm{Te}<973 \mathrm{~K})$. It has been established that at $\mathrm{Te}>973 \mathrm{~K}$, cylindrical particles ignite faster than cubic particles, at higher temperatures the situation is diametrically opposite (the cylinders are ignited earlier than the cubes).

The work was supported by the scientific schools grant NSH-7538-2016.8.

\section{References}

1. U. Mantau, Biomass and bioenergy, 79 (2015)

2. U. Mantau, U. Saal, K. Prins, F. Steierer, M. Lindner, H. Verkerk, Real potential for changes in growth and use of EU forests. EUwood (University of Hamburg Centre of Wood Science, Hamburg, 2010)

3. H. Weimar, P. Doring, U. Mantau, Einsatz von Holz in BiomasseGrobfeuerungsanlagen 2011, Final report (University of Hamburg Centre of Wood Science, Hamburg, 2012)

4. P. Lauri, P. Havlik, G. Kindermann, N. Forsell, H. Bottcher, M. Obersteiner, Energy Policy, 66 (2014)

5. Fuel and Energy Abstracts. 37, (96) 05114 (1996)

6. Y. Voytenko, P. Peck, Biomass and bioenergy, 38 (2012)

7. L.J.R. Nunes, J.C.O. Matias, J.P.S. Catalao, Renewable Energy, 85 (2016)

8. C. McGlade, J. Speirs, S. Sorrell, A review of regional and global estimates of unconventional gas resources (A Report to the Energy Security Unit of the Joint Research Centre of the European Commission, London, 2011)

9. T. Klason, X.S. Bai, Fuel, 86 (2007)

10. C. Antwi-Boasiako, B.B. Acheampong, Biomass and Bioenergy, 85 (2016)

11. H. Carslaw, J. Jaeger, Conduction of Heat in Solids (Oxford University Press, Oxford, 1959)

12. S.V. Syrodoi, G.V. Kuznetsov, V.V. Salomatov, Solid Fuel Chemistry, 49 (2015)

13. V.V. Salomatov, G.V. Kuznetsov, S.V. Syrodoy, N.Y. Gutareva, Appl. Therm. Eng. 106 (2016) 